\title{
Ionic interactions: comparative topological approach
}

\author{
Julia Contreras-García ${ }^{\mathrm{a}, \mathrm{b}, \mathrm{c}, *}$, Mónica Calatayud ${ }^{\mathrm{a}, \mathrm{b}, \mathrm{d}}$, Jean-Philip Piquemal ${ }^{\mathrm{a}, \mathrm{b}}$, \\ J.M. Recio ${ }^{\mathrm{c}, \mathrm{e}}$ \\ ${ }^{a}$ UPMC Univ Paris 06, UMR 7616, Laboratoire de Chimie Thorique, F-75005, Paris, France \\ ${ }^{b}$ CNRS, UMR 7616, Laboratoire de Chimie Thorique, F-75005, Paris, France \\ ${ }^{c}$ MALTA-Consolider Team, Spain \\ ${ }^{d}$ Institut Universitaire de France, France \\ ${ }^{e}$ Dpto. de Química-Física y Analítica, Universidad de Oviedo, E-33006 Oviedo, Spain
}

\begin{abstract}
The non covalent interaction (NCI) index applicability is extended in this paper to include ionic interactions, thus confirming its ability to detect both electronic and electrostatic effects. The characteristics of the reduced density gradient $v s$ the density, $s(\rho)$, and its isosurfaces are modeled on the basis of a promolecular model, enabling the topological features of the function in ionic compounds to be thoroughly understood. The NCI analysis to ionic compounds is extended to periodic systems in order to shed light on solid-state interactions. NCI reveals that anion-anion interactions contribute to crystal stability. These characteristics are compared to complementary methodologies, such as AIM and ELF, providing a quantitative framework for their comparison through the validity of the promolecular model. Shifts between nonhomeomorphic density and ELF topologies are shown to be related to the relative hardness of ions.
\end{abstract}

Keywords: noncovalent interactions, topological analysis, ionic bonding, ELF, AIM, NCI

\section{Introduction}

Molecular structure is governed by covalent and noncovalent interactions. However, it is mainly noncovalent interactions that are the driving force behind most biochemical processes. Chemical interactions between a protein and a drug or a catalyst and its substrate, the self-assembly of nanomaterials [1, 2], and even some chemical reactions $[3,4]$ are dominated by noncovalent interactions. These interactions include all interactions that are not due to electron sharing and they span a wide range of binding energies and encompass hydrogen bonding, electrostatic interactions, steric repulsion, and London dispersion [5].

\footnotetext{
* Corresponding author

Email address: contrera@lct.jussieu.fr (Julia Contreras-García)
} 
Noncovalent interactions might arise from two grounds: electronic (interference) or electrostatic (due to permanent or fluctuating charges).

More specifically, electrostatic interactions play a central role in a variety of biological processes. At any given $\mathrm{pH}$, proteins have charged groups that may participate in binding, both in their folding and self-assembly as well as in their binding to other molecules. For example, negatively-charged carboxyl groups on aspartic acid (Asp) and glutamic acid (Glu) residues may be attracted by the positively-charged free amino groups on lysine (Lys) and arginine (Arg) residues. Electrostatic effects exert strongly stabilizing influences on cations, in many cases controlling the conformational preferences of these cations [6]. The lowest energy conformers are those where the positive charge is brought closest to substituents bearing partial negative charges. These conformational biases, along with stereoelectronic effects, can control the stereoselectivity of reactions involving carbo-cationic intermediates.

Noncovalent interactions, not being related directly to the molecular structure but to its voids, still give rise to vigorous debates about their presence and strength. Graphic visualization of macromolecular structures is having significant impact on the field of structure biology. Since electrostatic interactions are governed by the Coulomb law, these have most commonly been identified by distance analyses. However, this approach totally disregards electronic effects. It is thus of particular value to be able to identify the existence of both electronic and electrostatic interactions which determine the protein structure and folding on the same basis.

We have recently developed a new approach, known as NCI (for NonCovalent Interactions), to detect noncovalent interactions in real space, based on the electron density and its derivatives [7]. This approach provides a rich representation of electronic effects such as van der Waals interactions, hydrogen bonds, and steric repulsion. Most importantly, the method, solely requiring knowledge of the atomic coordinates, is efficient and applicable to large systems, such as proteins or DNA. Thus, the extension to include ionic interactions would be extremely appealing for the understanding of biomolecules. In this paper, the abilities of this approach will be taken one step further to include ionic bonding, thus covering the whole range of noncovalent interactions (electronic and electrostatic) and introducing a holistic tool that can be also applied to large biosystems.

The paper is organized as follows. Firstly, we will give an overview of the topological tools that will be used in this contribution (AIM, ELF, NCI). Previous topological analyses (AIM, ELF) of ionic bonding will be introduced. Secondly, the computational details will be reviewed. In the Results section, we will: (i) show how electrostatic interactions engross the list of interactions described by NCI; (ii) show the effects of periodicity in ionic crystals; (iii) establish a quantitative comparison between the AIM-ELF-NCI interpretatins of ions and their radii. The paper ends with a summary of the most important findings of the paper. 


\section{Theoretical background}

\subsection{Topological analysis}

Over the years, different approaches have been developed to understand chemical bonds. Molecular Orbital (MO) theory has been very useful and successful for the theoretical analysis of chemical reactions and chemical reactivity. Frontier orbital theory [8] and the orbital symmetry rules of Woodward and Hoffman [9] are paradigmatic examples of the possibilities of quantum chemistry within MO theory. To reduce the dimensionality of the problem, threedimensional interpretative approaches have been put forward. The conceptual density functional theory pioneered by R.G. Parr [10] has been at the origin of such useful reactivity descriptors.

Another low dimensional approach has been pioneered by Bader [11]: the topological approach. Topological analysis relies on a partition of the molecular space achieved in the framework of the theory of gradient dynamics applied to a scalar potential function, say $V(\vec{r})$, which contains the physical or chemical information that we shall call "chemical potential function". Two types of topological information can be collected: local and global. Local information is gathered by evaluating density properties at distinctive points where the gradient vanishes. These points constitute a subset of special points of the field, named critical points (CPs), which contrary to wandering points $(\nabla V \neq 0)$, may act as a source or a sink of more than one gradient path, depending on their nature. The number of nonzero eigenvalues of the Hessian, or rank, determines its dimensionality, and the sum of the signs of the Hessian eigenvalues characterizes the capability to receive or send gradient paths. In a stable 3D topology, this gives rise to the following possibilities: $(3,-3)$ for local maxima or attractors, $(3,+3)$ for local minima, and $(3,-1)$ and $(3,+1)$ for saddle points in one or two dimensions, respectively.

A region of space can be associated with each attractor or sink of the field. This partitioning gives rise to a set of non-overlapping volumes or basins, $\Omega_{i}$, whose boundaries or separatrices are zero-flux surfaces. Global information may then be acquired from volume integration of property densities over these spatial fragments. Most commonly, the integration of the density, $\rho$, over their volume, $\Omega_{i}$, assigns a population, $\bar{N}$, to each basin:

$$
\begin{array}{r}
\bar{V}\left(\Omega_{i}\right)=\int_{\Omega_{i}} d \vec{r} \\
\bar{N}\left(\Omega_{i}\right)=\int_{\Omega_{i}} \rho(\vec{r}) d \vec{r}
\end{array}
$$

\subsection{Chemical potential functions}

Depending on the function used for the topological analysis, different partitions and chemical information can be retrieved from the chemical system. Since the potential functions used in the partition have a chemical meaning, the global properties (charges and volumes) so obtained also have a chemical meaning. For example, integration of the density within the AIM framework 
Table 1: Comparison between AIM, ELF and NCI topologies in benzene dimer

\begin{tabular}{l|ccc}
\hline Method & AIM & ELF & NCI \\
\hline \hline Function & Electron density & Pauli kinetic energy density & Reduced density gradient \\
Partition & Atoms & Lewis' pairs & Non covalent interactions \\
& & &
\end{tabular}

provides atomic charges [11], whereas integration of the density within ELF regions $[12,13]$ will yield information on the charge associated with bonds and lone pairs [14].

Three complementary chemical potential functions will be used in this paper, which are summarized in Table 1:

- The electronic density.

In the Atoms In Molecules (AIM) theory of Bader, the scalar function is the electron density distribution, whose basins have their attractors most commonly located on the nuclei and which are therefore associated with the atoms that constitute the molecule. According to Bader [11], the presence of a $(3,-1)$ critical point of the density is a necessary condition for the two atoms to be bonded to one another when the system is in a stationary state (thus, $(3,-1)$ critical points are called bond critical points within this framework).

The example in Table 1 shows the ability of AIM to identify the bonds in benzene dimer, both inter- and intramolecular. Bond critical points (in red) appear between covalently bonded $\mathrm{C}-\mathrm{C}$ and $\mathrm{C}-\mathrm{H}$ pairs as well as for the intermolecular weak interactions. Ring critical points (yellow) appear associated with the benzene ring as well as with the intermolecular interaction. As we will see, these ring critical points will also be critical points in the NCI topology. However, the fact that interactions are related to saddle points, locates them on the interatomic surfaces, so that they highlight interatomic contact, but they do not have an associated region within this approach.

- The kinetic energy density.

In order to recover a chemist's representation of a molecule consistent with Lewis' valence picture, one must use another local function that is able to describe the electron pair regions. The Electron Localization Function (ELF), $\eta$, was originally designed by Becke and Edgecombe to identify "localized electronic groups in atomic and molecular systems" [15]. As 
interpreted by Savin [16], the ELF core, $\chi$, can be understood as a local measure of the excess of local kinetic energy of electrons due to the Pauli principle, $t_{p}$ :

$$
\chi_{\sigma}(\vec{r})=\frac{t_{p}(\vec{r})}{t_{H E G}(\vec{r})}
$$

in which is $t_{p}$ is the difference between the definite positive kinetic energy $t(\vec{r})$ and the von Weizsäcker kinetic energy functional:

$$
t_{p}(\vec{r})=t(\vec{r})-\frac{1}{4} \frac{\left|\rho_{\sigma}(\vec{r})\right|^{2}}{\rho_{\sigma}(\vec{r})}
$$

and $t_{H E G}(\vec{r})=\frac{3}{5}\left(6 \pi^{2}\right)^{2 / 3} \rho_{\sigma}^{5 / 3}(\vec{r})$ is the kinetic energy density of the homogeneous electron gas.

ELF values are confined in the $[0,1]$ range by a Lorentzian transformation which facilitates its interpretation:

$$
\eta=\frac{1}{1+\chi^{2}}
$$

ELF basins appear associated with localized electron pairs, such as bonds, lone pairs and atomic shells, thus matching the VSEPR model. As for noncovalent interactions, changes are observed in the ELF value at the first order saddle point $[17,18]$. This approach, known as the Electron Localizability Indicator (ELI), enables bond formation and delocalization to be understood from one electron properties. However, due to the lack of electronic localization in most noncovalent interactions, this approach is not able to visualize weak interactions. As can be seen in Table 1, the VSEPR regions of benzene dimer are clearly identified by ELF, but nothing is seen for the interbenzene stacking interaction.

- The reduced density gradient.

NCI is a visualization index based on the analysis of the reduced density gradient, $s$, at low densities [7, 19]:

$$
s=\frac{1}{C_{F}} \frac{|\nabla \rho|}{\rho^{4 / 3}}
$$

where $c_{F}=2\left(3 \pi^{2}\right)^{1 / 3}$ is the Fermi constant and the $4 / 3$ exponent in the density ensures that $s$ is a dimensionless quantity. Figure 1 shows the reduced density gradient vs electron density for formic acid (Fig. 1a) and formic acid dimer (Fig. 1b). These graphs have been obtained with the NCIPLOT code written by some of the authors [19]. This code constructs a grid over the molecular space, analyzing $\rho$ and $s$ point-wise, which enables the reconstruction of the reduced density gradient behavior as shown in Figure 1. This representation shows characteristic peaks at low densities in the presence of noncovalent interactions due to the annihilation of the density gradient at these points (Fig. 1b). The isosurfaces that encom- 


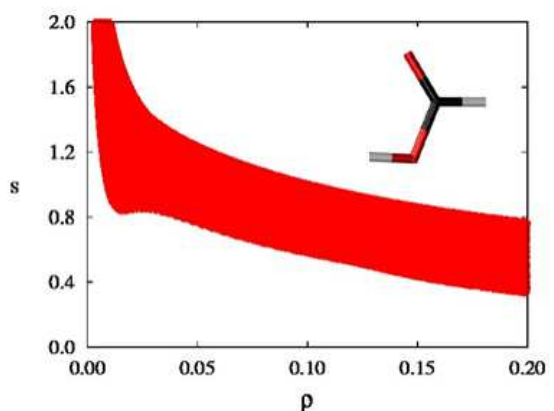

(a)

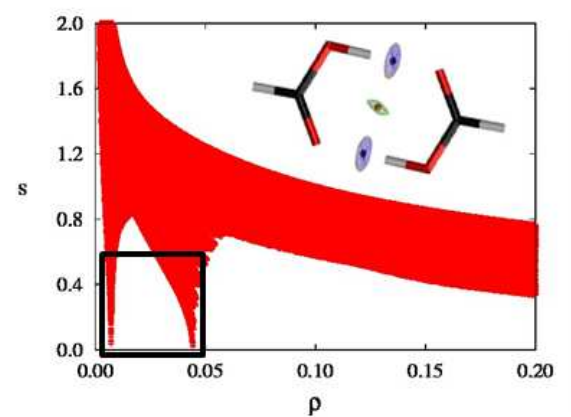

(b)

Figure 1: NCI analysis in the formation of formic acid dimer. (a) $s(\rho)$ for formic acid (b) $s(\rho)$ for formic acid dimer. NCI isosurfaces are shown in the insets for $s=0.6$ a.u. and colored according to a BGR scheme over the range $-0.04<\operatorname{sign}\left(\lambda_{2}\right) \rho<0.4$ a.u. Critical points of the density have been included for comparison and are colored according to their signature: $(3,-1)=$ black, $(3,+1)=$ red.

pass these interaction-peaks contour the noncovalent interactions in the system, both favorable and unfavorable. As an example, Table 1 shows both the steric repulsion associated with the ring tension and the attractive dispersive interaction between the benzene monomers. In order to differentiate between these different types of interactions, the sign of the second density Hessian eigenvalue times the density is used. This value is able to characterize the strength of the interaction by means of the density, and its curvature by means of the sign of the second eigenvalue.

This method is applicable to small molecules as well as inorganic complexes. Furthermore, the method is also applicable to promolecular densities, enabling the analysis of biomolecules.

\subsection{Ionic bonding - topological approach background}

Probably the simplest and earliest proposed classification of bonding within the theory of Atoms In Molecules [11] is that based on the sign of the laplacian at the bond critical point, $\nabla^{2} \rho_{(3,-1)}=\lambda_{1}+\lambda_{2}+\lambda_{3}$, where $\lambda_{i}$ are the main curvatures. Since $\lambda_{3} \geq \lambda_{2} \geq \lambda_{1}$, we have that $\lambda_{3}>0$ and $\lambda_{2}, \lambda_{1}<0$ at the bond critical point. Generally, shared chemical interactions (covalent and polar bonds) are characterized by negative $\nabla^{2} \rho_{(3,-1)}$ values and hence dominant negative curvatures, with lowering of the potential energy arising from the charge being concentrated between the nuclei along the bond path. Conversely, closedshell interactions (ionic bonds, hydrogen bonds, van der Waals interactions) have positive $\nabla^{2} \rho_{(3,-1)}$ values and hence a dominant positive $\lambda_{3}$ curvature, with their energetic stability being attained from the electronic charge that is concentrated within the atomic basins, rather than being shared between them. Closed-shell interactions are characterized by relatively low values of $\rho_{(3,-1)}$ because the density contracts away from the interatomic surface in requirement of 
the Pauli exclusion principle. In the case of ionic interactions, a further charge transfer is observed after integration of the corresponding basins [20].

It should be noted that ionicity is most clearly observed in the solid state, arising from the stabilization of charges throughout the periodic network. Within the framework of the topology of the electron density, an in-depth comprehensive analysis of ionic crystals has been carried out by Luaña et al. [21] and Pendás et al.[22, 23].

Although the AIM approach enables the strength of an ionic bond to be ascertained, it does not provide insight into the bonding in terms of electronic pairing within the crystal. The ELF function provides further insight in this direction. From the advent of the visual ELF analysis [24], it was recognized that ionic compounds were identified within this framework by a closed-shell electrostatic interaction between ions, which in the ELF approach involves the lack of bonding basins, only showing closed-shell basins whose shape approach that of a sphere [25].

Indeed, due to the absence of bonding basins, not much attention was paid initially to the topological analysis of ELF in ionic crystals. This gap was even wider for solids, where the computational complexity of the localization of all the critical points restricted its full exploitation. By means of a code $[26,27]$ recently implemented by the authors, these difficulties have been overcome, enabling determination of bond formation and delocalization between basins by means of the analysis of $\operatorname{ELF}(3,-1)$ critical points $[17,18]$. As far as ionic interactions are concerned, a comprehensive and quantitative description of ionic solids within the ELF framework has recently been introduced, showing how coordination and phase transitions can be tracked by means of the complete determination of the ELF topology [25, 28, 29].

\section{Computational details}

To obtain plots of the electron density and reduced density gradient densityfunctional in molecules, calculations were performed with the B3LYP functional $[30,31]$ and the $6-31 \mathrm{G}^{*}$ basis set, using the Gaussian 09 program [32]. Molecular geometries were optimized at the same level through the Berny algorithm [32, 33]. Plots of $s$ versus $\rho$ were obtained from the NCIPLOT program developed by some of the authors $[19,34]$ with grids of 0.1 Aalong each axis.

In solids, crystalline wavefunctions were obtained for the B1 phase of the AX (A: Li, Na, K; X: F, Cl, Br) alkali halide family within the density functional theory framework under the generalized gradient approximation with Becke exchange [35] and PW91 correlation [36] functionals as implemented in the CRYSTAL98 code [37]. Basis sets were taken from literature sources [38, 39] and unit cell parameters were set to the experimental values of the stable rock-salt structures at room temperature conditions [23].

AIM, ELF and NCI visualization for solids was implemented in a second version of the critic code [40], which can use density information from Quantum ESPRESSO [41], abinit [42, 43], VASP [44, 45], WIEN2k [46-48], elk [49] or pi7 

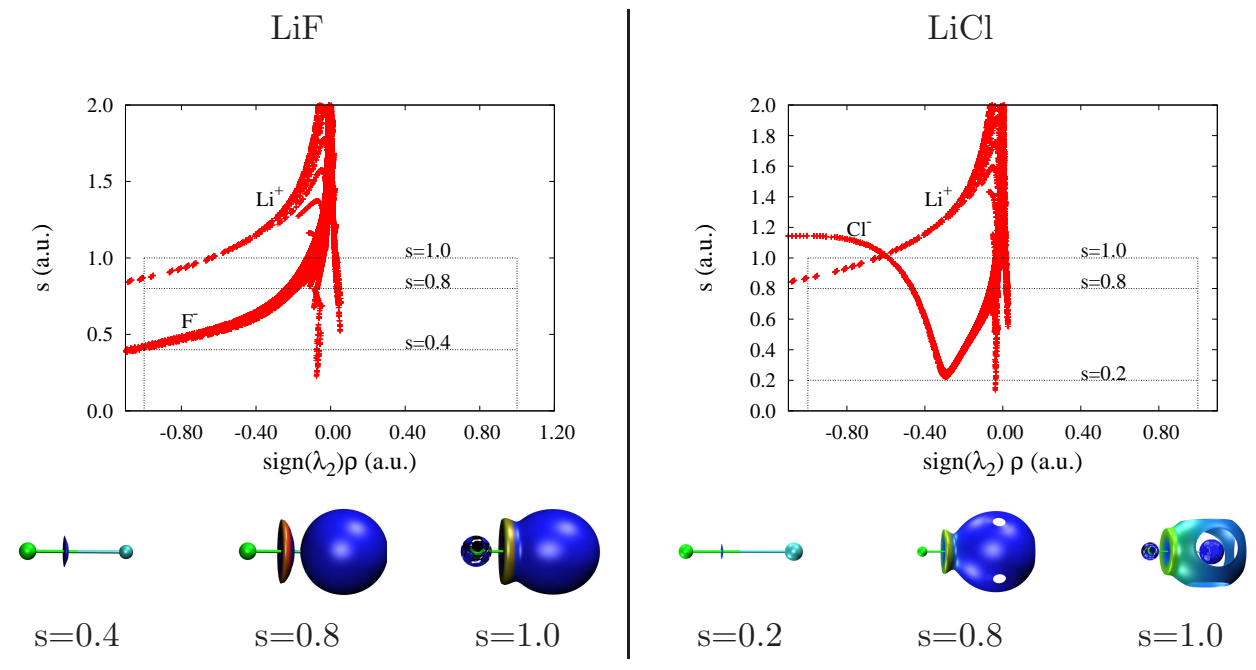

$\mathrm{s}=0.2 \quad \mathrm{~S}=0.8 \quad \mathrm{~S}=1.0$

Figure 2: NCI for LiF (left) and $\mathrm{LiCl}$ (right). Top: $s(\rho)$, Bottom: NCI isosurfaces for various cutoffs (indicated below each figure). The part of the $s(\rho)$ diagram covered by each isosurface is highlighted in the $s(\rho)$ plot by the corresponding frames.

[50] as input. In addition, critic2 reads density cubes in Gaussian [32] and XCrysden [51] formats, so any program not directly supported can be interfaced with critic2 via density grids.

\section{Results \& Discussion}

\subsection{Ionic bonding within NCI}

The following set of ionic molecules were analyzed LiX, NaX and KX, with $\mathrm{X}=\mathrm{F}, \mathrm{Cl}$. The results of this analysis are collected in Figs 2-4. Several common characteristics arise: two different decaying curves at different heights (some with a change in slope at medium densities) and a set of two nearly symmetrical peaks.

Densities in ionic crystals are almost non overlapping, thus they are well described by promolecular densities [23]. Atomic/ionic densities can be modeled by exponential basis functions for each shell $[52,53]$ :

$$
\rho_{a t}^{p r o m}=\sum_{n} c_{n} e^{\zeta_{n} r}
$$

where $n$ stands for the number of shells. In the region of the interaction, this reduces to the valence (i.e. the outermost shell), so that the density for an ionic pair $\mathrm{AB}$ at a mutual distance $R$ is given by:

$$
\rho(r)=\rho_{A}^{p r o m}+\rho_{B}^{p r o m}=a e^{-\alpha r}+b e^{-\beta(R-r)}
$$

where $a, \alpha, b$ and $\beta$ are positive constants characteristic of the A and B ions, respectively [23]. 

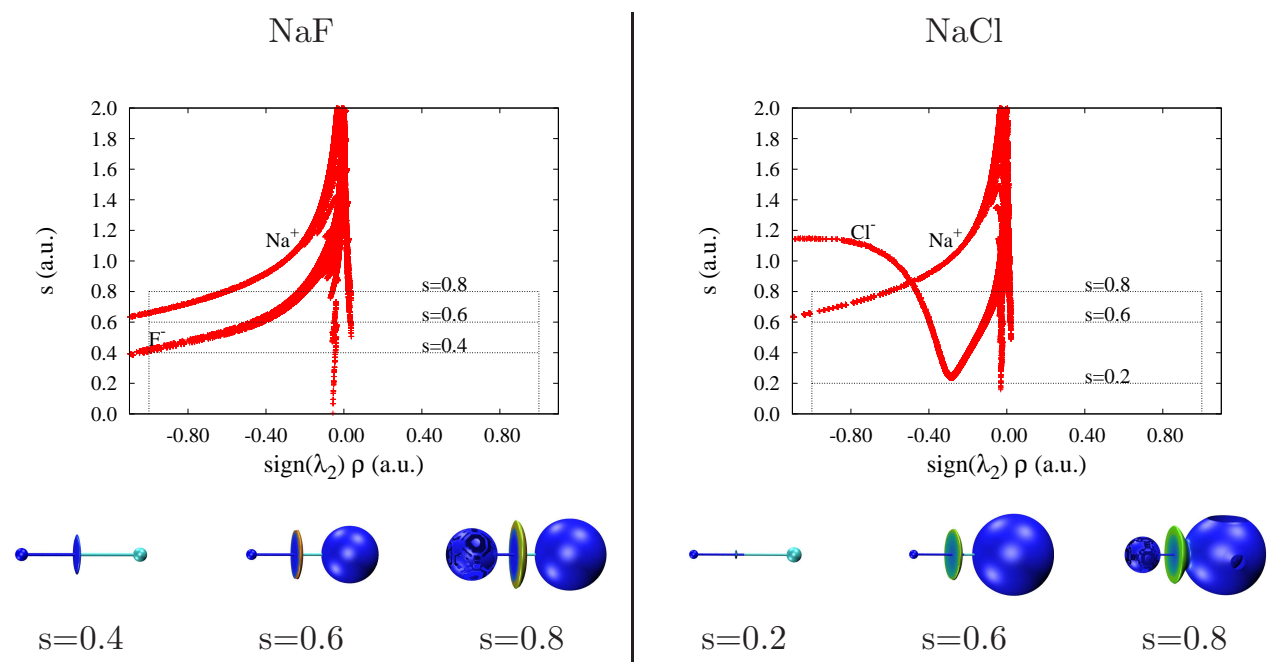

Figure 3: NCI for NaF (left) and $\mathrm{NaCl}$ (right). Top: $s(\rho)$, Bottom: NCI isosurfaces for various cutoffs (indicated below each figure). The part of the $s(\rho)$ diagram covered by each isosurface is highlighted in the $s(\rho)$ plot by the corresponding frames.

For $r$ close to $\mathrm{A}$, we can assume that $\rho(r) \simeq \rho_{A}(r)=a e^{-\alpha r}$, and analogously for $r$ close to B. In these cases, the reduced density gradient reduces to the freeion behavior:

$$
s\left(r \rightarrow r_{A}\right)=\alpha a e^{-\alpha r / 3}=\alpha \rho^{-1 / 3}
$$

Thus, these regions give rise to two curves of a $y=c x^{-1 / 3}$ exponential behavior, where the constant $c$ determines the displacement of the curve along the $y$-axis. The exponential constants $\alpha$ and $\beta$ are associated with the difficulty of the ions to deform, i.e. with their hardness [23]. Thus, the relative position of the curves can be associated with the relative hardness of the ions.

Since cations are generally harder than anions, i.e. $\alpha>\beta$, in most cases the cation curve will be over the anionic line at low (valence) densities. This is exactly what is observed in LiX (Fig. 2 top) and NaX (Fig. 3 top), with $\mathrm{X}=\mathrm{F}, \mathrm{Cl}$ : two decaying curves, one for each ion, with the cation on top of the anion by $\alpha-\beta$. However, for potassium salts, the cation is softer, even yielding an inversion for $\mathrm{KF}$ (Fig. 4 left), where the anionic $\mathrm{F}^{-}$curve is over the $\mathrm{K}^{+}$ line at low densities. Indeed, the similar hardness of $\mathrm{K}^{+}$and $\mathrm{F}^{-}$was already observed within the AIM [23] and ELF [25] topologies.

This promolecular analysis provides us with an easy to visualize way to recognize cation-anion contributions to the $s(\rho)$ plot as well as to visuliaze the relative hardness of the ions: the greater the difference between the curves, the greater the ionic hardness difference between them.

In the case of third row elements $(\mathrm{K}, \mathrm{Cl})$, a sudden change in slope is observed at medium densities. This observation can be easily understood by means of our promolecular model (Eq. 7). These densities lack relaxation, but since the overlap in ionic crystals is very small, it can also be used to understand the 

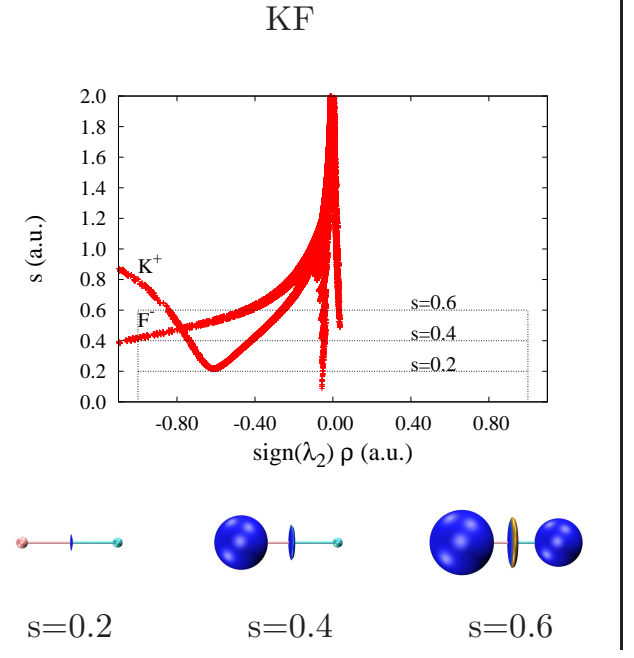

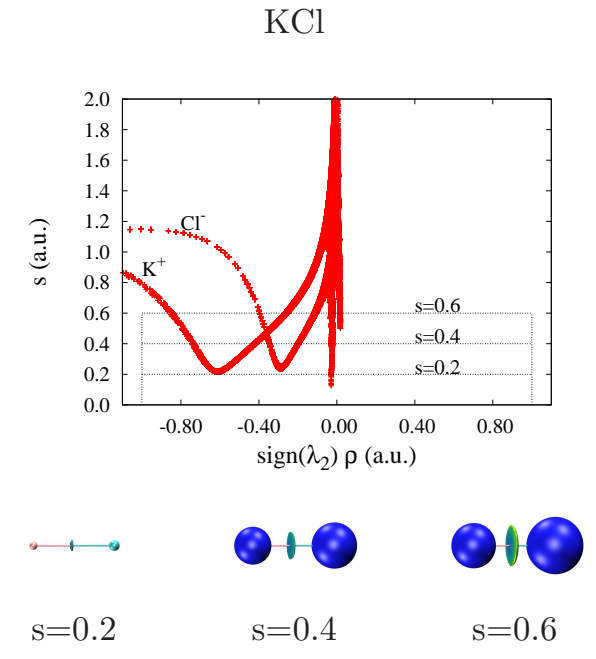

Figure 4: NCI for KF (left) and $\mathrm{KCl}$ (right). Top: $s(\rho)$, Bottom: NCI isosurfaces for various cutoffs (indicated below each figure). The part of the $s(\rho)$ diagram covered by each isosurface is highlighted in the $s(\rho)$ plot by the corresponding frames.

behavior of the $s(\rho)$ curve at the atomic level.

Fig. 5 shows the promolecular model for the chlorine density with the slope change observed in Figs 2-4 highlighted in the inset. The parameters were taken from Ref. [7]. When the behavior of each shell contribution was analyzed, it became clear that the change in slope is associated with a change from one shell to another with a different characteristic hardness ( $\zeta_{n}$ exponent).

Finally, the appearance of peaks in Figs. 2-4 is directly related to the existence of density $(3,-1)$ critical points between the ions. When the density gradient is zero, $s(\rho)$ also becomes zero (see Eq. 6). Since $s \geq 0$, the critical points of the density are minima of the reduced density gradient. The ionic bond critical point induces a crucial change in the reduced density gradient between the ions involved. Since the behavior of $s$ at low densities is dominated by $\rho \rightarrow 0$, it tends to $\infty$ except in the regions around a density critical point, where $\nabla \rho \rightarrow 0$ dominates, giving rise to a steep peak that goes to zero. The neighboring points of the density bond points show the same signature as the $(3,-1)$ critical point itself, giving rise to a negative second eigenvalue. However, as has already been observed in hydrogen bonds [54], the region surrounding the critical point shows the electronic repulsion induced by the density overlap, giving rise to $\lambda_{2}>0$. This is why two peaks are observed in the top Figs 2-4: one for $\lambda_{2}>0$ and another for $\lambda_{2}<0$.

The cutoff used in the representation of NCI isosurfaces will determine which features are observed. Due to the ionic critical points present in our systems, these noncovalent interactions will be observed from the beginning (Figs 2-4 bottom left). Disc-shaped isosurfaces appear in between the ions that represent the ionic bond. Thus, NCI is able to reveal all types of noncovalent bonds, 


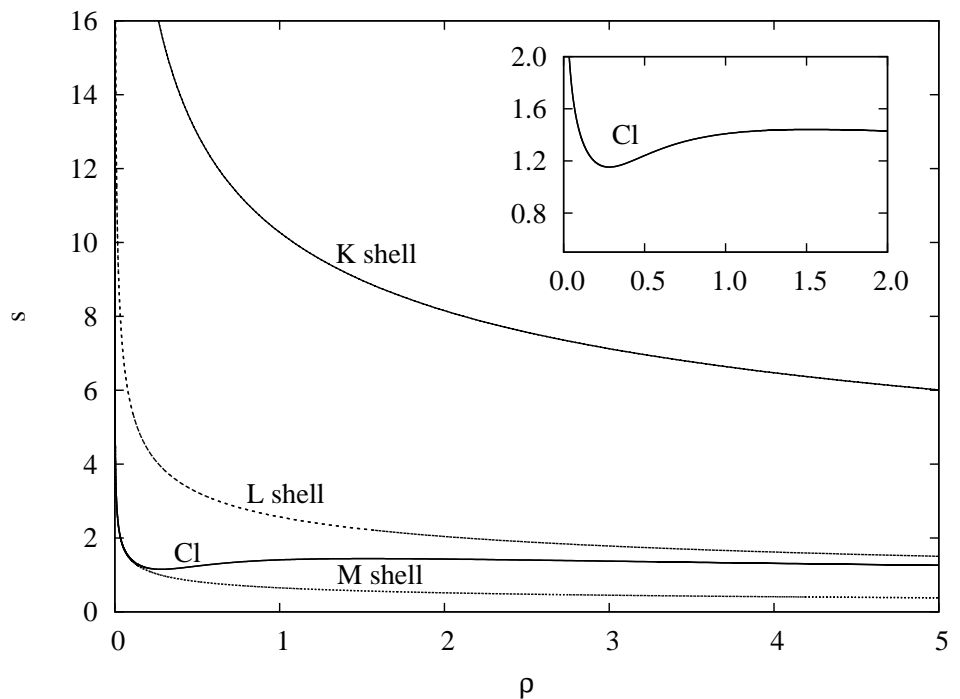

Figure 5: Promolecular model for $\mathrm{Cl}$ atom. Each shell is represented by an exponential as in Eq. 7, yielding 3 shell lines (K,L,M) for chlorine. The slope change observed in the ionic plots is highlighted in the inset. It is due to the fact that those densities are mainly dominated by the $\mathrm{L}$ shell and not anymore by the M shell.

be them electronic or electrostatic in origin. The size of the isosurfaces will be greater for fluorides than for chlorides, due to the fact that the peaks for the former have a greater surface than the latter. The coloring of the surface, determined by the density, will be related to the strength of the interaction.

The presence of repulsive areas in the NCI ionic interaction is visualized at greater cutoffs, when the region around the critical point is included. In the fluorides, where the density is stronger, the repulsion is easily seen as a red circle surrounding the bonding area. However, for greater anions, this repulsion is smaller, and only green surfaces are observed.

\subsection{Ionic interactions in crystals}

There are several factors that draw a clear line between the molecular and the crystalline realms as far as the study of their topology is concerned. Firstly, due to the periodicity of the solid, an infinite number of critical points is expected $[22]$.

Furthermore, for a periodic system, the Morse relationship that holds reads as follows:

$$
n_{(3,-3)}-n_{(3,-1)}+n_{(3,+1)}-n_{(3,+3)}=0
$$

$n$ being the number of critical points of a given type. The existence of $(3,+3)$ points is ensured by the Weierstrass theorem, which accordingly enforces the presence of the whole set of types of critical points of rank 3 within the following 


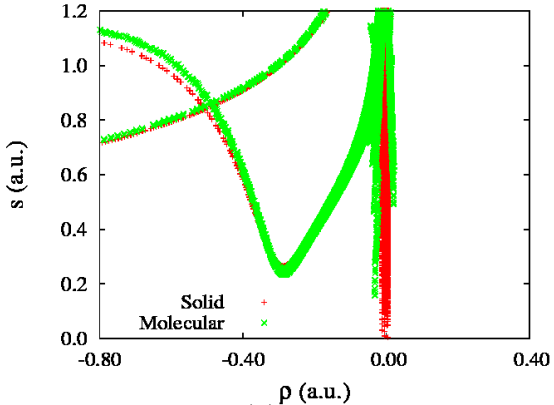

(a)

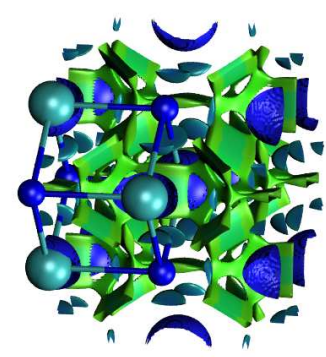

(b)

Figure 6: NCI for solid NaCl. a) $s(\rho)$ for solid (red) and molecular (green) NaCl, (b) NCI isosurface for $\mathrm{s}=0.5$.

lower limits:

$$
n_{(3,-3)} \geq 1, \quad n_{(3,-1)} \geq 3, \quad n_{(3,+1)} \geq 3, \quad n_{(3,+3)} \geq 1
$$

Figure 6 shows how NCI behaves in a simple crystal (fcc- $\mathrm{NaCl}$ ). It can be seen that the main $s(\rho)$ characteristics are preserved from the molecular realm. However, two slight differences appear, which are consistent with the known real-space features of the electron density. Firstly, the $s(\rho)$ plot becomes thinner. This is due to the highly symmetric pattern of the periodic density in the B1 phase. Secondly, new interactions appear related to the fact that all types of critical points should be present. These naturally appear at lower densities, giving rise to a continuous transition between the attractive and repulsive cationanion peaks. These interactions are related to dispersive interactions within the crystal, but most importantly to the anion-anion interactions (see Fig. 6) that were already observed both within the AIM [23] and the ELF [25] frameworks. Indeed, the occurrence of bonding interactions in a crystal between like-charged species that contribute significantly to the crystal packing formation has been observed, and debated, many times [23, 25, 55] and is here once again confirmed by NCI analysis. The strongest interaction in terms of electron density at the NCI domain is still the cation-anion contact.

\subsection{Quantitative comparative topological approach to ionic bonding}

The aim of this final subsection is to study the topological radii resulting from the AIM, ELF and NCI analysis of the alkali halides. Ionic radii have played a determinant role in solid state physics as the main structural parameter available to systematize crystal properties [56]. For a long time these distances were considered as the contact radii between spheres, regardless of the obvious drawbacks this hypothesis had. On the one hand, it is necessary to split the internuclear distance of adjacent atoms into contributions of different species. This requires an initial assignment of a reference radius for the construction of a congruent set of data. On the other hand, the spherical atomic 
volumes calculated from these radii do not reconstruct the cell volume. These two shortcomings are overcome when topological radii are used, since they are rigorously defined, and being direction dependent, they fill up the whole space. It is interesting to note that the existence of nonspherical ions is supported by experiments: recent measurements of accurate electron densities have proved that an ion may display different radii along different directions [57].

Bader's theory of Atoms In Molecules [11] provides an unambiguous framework in which to assign an accurate meaning to geometric coordinates in solids. In a condensed phase, an AIM-defined atom (or ion) is a closed region of the physical space, having well-defined boundaries and volume. The location of the ionic bond critical point between cations and anions allows defining both cationic and anionic radii within the crystal. In Section 4.1, we have seen that all critical points of AIM constitute minima of the NCI topology. Thus, the definition of ionic radii within both topologies is equivalent. This is, however, not the case of ELF, whose topology has been shown to be nonhomeomorphic with the density [58]. Within the ELF framework, the absence of bonding basins in ionic systems enables ionic superbasins to defined as the sum of core and valence shells belonging to the same ion. This allows the partition of the crystal into ions, whose radii are determined by the position of the $(3,-1)$ points along the cation-anion interaction line.

In order to quantitatively compare these topologies, density and ELF radii were taken from the literature (see Ref. [25] for computational details) for the B1 phase of the AX (A: Li, Na, K; X: F, Cl, Br) alkali halide family. Results for these topological radii are collected in Fig. 7, along with empirical radii. Topological radii are compared with two empirical sets of radii: Wasastjerna's [59] and Pauling's [60]. The former were determined from polarizabilities, whereas the latter are inversely proportional to the effective nuclear charge.

We can see that topologically-determined radii closely follow the overall empirical trends, both in anions and cations (Fig. 7). However, a slight deviation clearly arises. In both density and ELF topologies, anions are slightly smaller and cations are slightly larger. As already noted before [25], this deviation is more pronounced in the case of the AIM approach, with ELF radii always matching the experimental values closer.

The promolecular model previously introduced can be used to understand the relationship between the different sets of topological radii. In order to achieve this, we need to study the location of the points where the gradient of the density and the ELF annihilate along the bonding line: $\vec{r}_{\rho}$ where $\nabla \rho\left(\vec{r}_{\rho}\right)=0$ and $\vec{r}_{\eta}$ where $\nabla \eta\left(\vec{r}_{\eta}\right)=0$, respectively. Since the actual formulation of ELF is a mapping of its core $\chi$ (Eq. 5), it is only necessary to find the critical points associated with this simpler function. Furthermore, since ions are nearly spherical, we will only consider the main contribution, that is, radial derivatives. Going back to Eq. 3, the ELF or $\chi$ radius is determined by the following condition:

$$
\left.\frac{d \chi(r)}{d r}\right|_{r_{\eta}}=\frac{d t_{p}(r)}{C_{F} \rho^{5 / 3}(r)}-\frac{5}{3} \frac{d \rho(r) t_{p}}{c_{F} \rho^{8 / 3}(r)}=0
$$




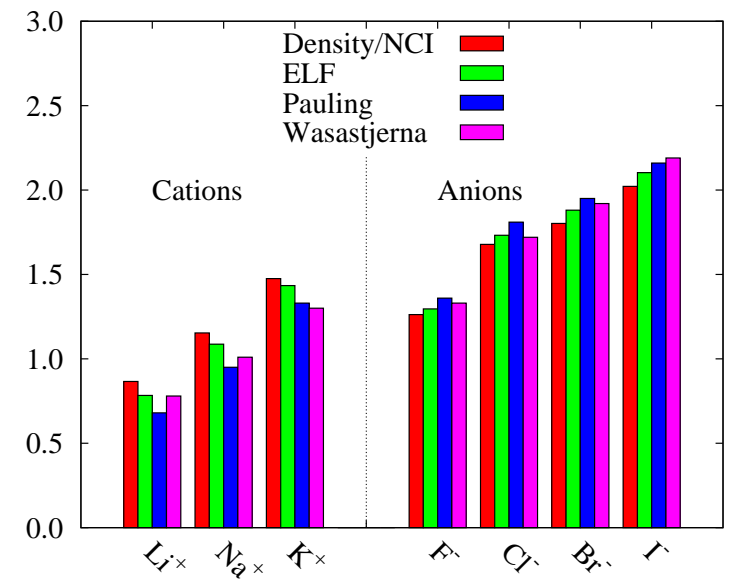

Figure 7: Ionic radii in the alkali halides according to ELF, Wasastjerna [59], Pauling [60] and QTAIM [23]. Atomic radii are also provided for comparison $(\AA)$.

After rearranging the terms, we obtain:

$$
\frac{d t_{p}(r)}{t_{p}(r)}-\frac{5}{3} \frac{d \rho(r)}{\rho(r)}=0
$$

where the first term, which we shall call $Y_{1}(r)=d t_{p}(r) / t_{p}(r)$, depends only on the kinetic energy density and its derivatives, and the second one, which we shall call $Y_{2}(r)=5 d \rho(r) / 3 \rho(r)$, depends only on the electronic density and its derivatives. This arrangement is especially interesting for inter-relating the position of the density and ELF critical points by means of the condition $Y=$ $Y_{1}-Y_{2}=0$.

Fig. 8 shows the behavior of $Y_{1}, Y_{2}$ and $Y$ for $\mathrm{LiF}$, with $\mathrm{Li}^{+}$on the left and $\mathrm{F}^{-}$on the right of the plot. The behavior of $Y_{2}(r)$ can be understood from the promolecular model. From Eq. 8 we can see that the density gradient and the laplacian are given by:

$$
\begin{array}{r}
\nabla \rho(r)=-\alpha a e^{-\alpha r}+\beta b e^{-\beta(R-r)} \\
\nabla^{2} \rho(r)=\alpha^{2} a e^{-\alpha r}+\beta^{2} b e^{-\beta(R-r)}>0
\end{array}
$$

Since $a, \alpha, b$ and $\beta$ are positive, the laplacian is positive everywhere and $\nabla \rho$ and $Y_{2}$ are increasing functions in ionic crystals which annihilate at the AIM critical point.

The position of the ELF critical point is hence shifted due to $Y_{1}(r)=$ $d t_{p}(r) / t_{p}(r)$. The sign of this contribution determines whether the shift takes place toward the cation or the anion. From its definition, the Pauli kinetic energy density, $t_{p}$, is positive. This can be seen in Fig. $8 \mathrm{~b}$, where we also see that $t_{p}$ increases toward the cation, yielding $d t_{p}<0$ in our case (cation is on 


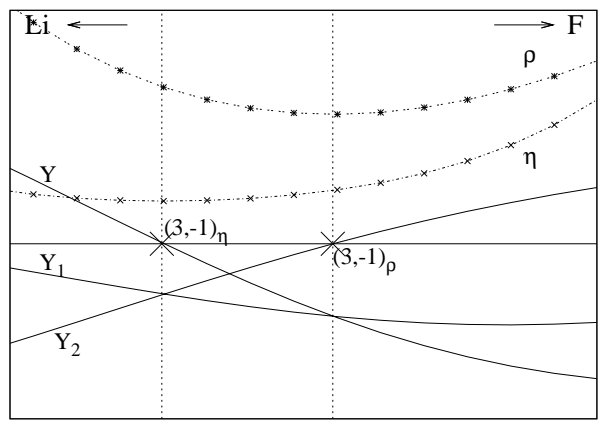

(a)

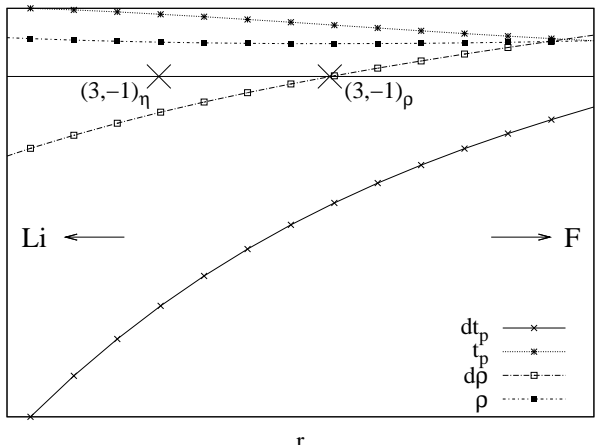

(b)

Figure 8: Decomposition of density and kinetic energy density terms of LiF around ELF, AIM and NCI critical points. $\mathrm{Li}^{+}$lies on the left and $\mathrm{F}^{-}$on the right. Values of $\rho$ and ELF have been rescaled for a clearer view. Meaning of symbols as in the text.

the right). Indeed, the sign of $d t_{p}$ can be deduced assuming that each ionic tail wavefunction can be reduced to one exponential $e^{-\alpha r / 2}=\rho_{A}^{1 / 2}$ and that close to the critical point, $\nabla \rho(r) \simeq 0$, so that $t_{p} \simeq t$, with:

$$
d t=\frac{\alpha^{3} a}{16} e^{-\alpha r / 2}-\frac{\beta^{3} b}{16} e^{-\beta(R-r) / 2}=\alpha^{3} \rho_{A}^{1 / 2}-\beta^{3} \rho_{B}^{1 / 2}<0
$$

where we have made use of the fact that most generally $\alpha>\beta$, so that both $d t$ and $d t_{p}$ are negative in our ionic arrangement. It follows that the kinetic energy density increases toward the harder ion as seen in Fig. 8b. Therefore, $Y_{1}$ is negative and shifts the curve toward the cation, thus explaining the fact that the ELF cationic radii are smaller than the AIM and NCI ones.

Fig. 8 illustrates how $Y_{1}(r)$ stays negative along the whole interacting region for LiF, giving rise to a shift of $Y(r)$ toward the cation. Hence, the ELF critical point, where $Y_{1}(r)=Y_{2}(r)$ arises closer to the latter.

It is important to note that there has always been a major concern in understanding the relationship between the topology induced by ELF and that induced by the electron density. Resorting to ionic topologies, where all chemical quantities are well defined (ions), has enabled us to make a quantitative comparison of these topologies, shedding light on the fact that the relative hardness of ions is responsible for the shift between ELF and AIM critical points. Indeed, greater differences are observed for harder cations (see $\mathrm{Li}^{+}$in Fig. 9) and softer anions (see $\mathrm{Cl}^{-}$and $\mathrm{Br}^{-}$in Fig. 9).

\section{Conclusions}

Graphic visualization of macromolecular structures is having significant impact on the field of structure biology. Since electrostatic interactions are gov- 


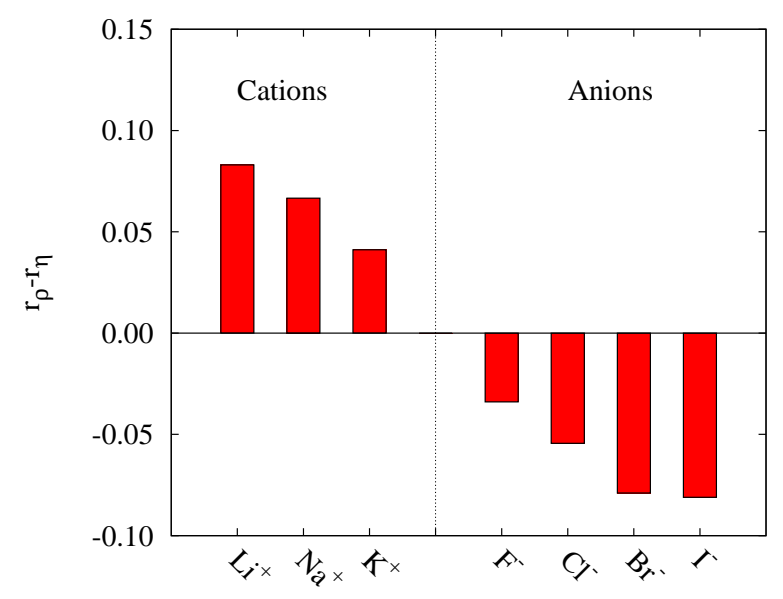

Figure 9: Difference between AIM and ELF radii in $\AA$ for cations and anions in the alkali halide family

erned by the Coulomb law, these have most commonly been identified by distance analyses. However, this approach totally disregards electronic effects.

In this contribution, we have put forward the basis for a combined analysis of electrostatic and electronic noncovalent interactions in terms of the NonCovalent Interaction index. The applicability of this index, used up to now only for electronic effects, has thus been enlarged to include ionic interactions. The behavior of this index in ionic compounds has been well characterized: the behavior of atomic tails has been analyzed in terms of the promolecular model and the peaks have been put in relationship with the AIM topology. The applicability of NCI to large biosystems, along with its ability to describe electronic and electrostatic effects, will be the subject of a future contribution, in which protein interactions will be analyzed.

A new code for crystalline systems has been developed in order to establish the differences introduced by periodicity. The existence of anion-anion interactions has been confirmed from the NCI point of view as contributing (though less than direct contacts) to crystal stability.

Finally, a new step has been taken in the understanding of the relationship between the topologies induced by different chemical potential functions. In this contribution, ionic compounds have been used as a prototypical example where both ELF and AIM topologies should yield similar results. The shift between the two has been quantitatively related to the relative hardness of the ions involved, thus taking a first step towards the quantitative understanding of the relationship between nonhomeomorphic topologies. 


\section{Acknowledgments}

Financial and computational support from the MALTA-Consolider Ingenio2010 program under project CSD2007-00045 is acknowledged. JCG thanks the Spanish Ministry of Education for a postdoctoral grant (Orden EDU/2253/2010). JMR thanks the Spanish Ministry of Education for financial support (CTQ200914596-C02-02). The authors thank G. ONeill for proof-reading the manuscript.

\section{References}

[1] H. Fenniri, M. Packiarajan, K.L. Vidale, D.M. Sherman, K. Hallenga, K.V. Wood, J.G. Stowell, J. Am. Chem. Soc. 123 (2001) 3854.

[2] P. Kruse, E.R. Johnson, G.A. DiLabio, R.A. Wolkow, Nano Lett. 2 (2002) 807.

[3] S.S. Sheiko, F.C. Sun, A. Randall, D. Shirvanyants, M. Rubinstein, H. Lee, K. Matyjaszewski, Nature. 440 (2006), 191.

[4] G.A. DiLabio, P.G. Piva, P. Kruse, R.A. Wolkow, J. Am. Chem. Soc. 126 (2004) 16048.

[5] P.A. Kollman, Chem. Rev. 10 (1977) 365.

[6] D.M. Smith, K.A. Woerpel, Org. Biomol. Chem. 4 (2006) 1195.

[7] E.R. Johnson, S. Keinan, P. Mori-Sánchez, J. Contreras-García, A.J. Cohen, W. Yang, J. Am Chem. Soc 132 (2010) 6498.

[8] K. Fukui, T. Yonezawa, H. Shinghu, J. Chem. Phys. 20 (1952) 722.

[9] R.B. Woodward, R. Hoffmann, Angew. Chem., Int. Ed. Engl. 8 (1969) 781.

[10] R.G. Parr, R.A. Donnelly, M. Levy, W.E. Palke, J. Chem. Phys. 68 (1978) 3801.

[11] R.F.W. Bader, Atoms in Molecules: A. Quantum Theory, Oxford University. Press, Oxford, 1990.

[12] B. Silvi, A. Savin, Nature 371 (1994) 683.

[13] U. Häussermann, S. Wengert, R. Nesper, Angew. Chem. Int. Ed. Engl. 33 (1994) 2069.

[14] M. Kohout, A. Savin, Int. J. Quantum Chem. 60 (1996) 875.

[15] A.D. Becke, K.E. Edgecombe, J. Chem. Phys. 92 (1990) 5397.

[16] A. Savin, O. Jepsen, J. Flad, L.K. Andersen, H. Preuss, H.G. von Schnering, Angew. Chem. Int. Ed. Engl. 31 (1992) 187. 
[17] J. Contreras-García, J.M. Recio, Theor. Chem. Acc. 128 (2011) 411.

[18] J. Contreras-García, A. Martín Pendás, B. Silvi, J.M. Recio, J. Phys. Chem. B. 113 (2009) 1068.

[19] J. Contreras-García, E.R. Johnson, S. Keinan, R. Chaudret, J-P. Piquemal, D. Beratan, W. Yang, J. Chem. Theory Comput. 7 (2011) 625.

[20] P. Mori-Sánchez, A. Martín Pendás, V. Luaña, J. Am. Chem. Soc 124 (2002) 14721.

[21] V. Luaña, A. Costales, A. Martín Pendás, Phys. Rev. B 55 (1997) 4285.

[22] A. Martín Pendás, A. Costales, V. Luaña, Phys. Rev. B 55 (1997) 4275.

[23] A. Martín Pendás, A. Costales, V. Luaña, J. Phys. Chem. B 102 (1998) 6937.

[24] A. Savin, R. Nesper, S. Wengert, T.F. Fässler, Angew. Chem. Int. Ed. Engl. 36 (1997) 1809.

[25] J. Contreras-García, J.M. Recio, J. Phys. Chem. C, 115 (2011) 257.

[26] J. Contreras-García, A. Martín Pendás, B. Silvi, J. M. Recio, J. Phys. Chem. Solids 69 (2008) 220.

[27] J. Contreras-García, A. Martín Pendás, B. Silvi, J.M. Recio, J. Theor. Chem. Comp. 5 (2009) 164.

[28] J. Contreras-García, A. Martín Pendás, B. Silvi, J.M. Recio, J. Phys. Chem. B. 112 (2008) 9787.

[29] J. Contreras-García, P. Mori-Sánchez, B. Silvi, J.M. Recio, J. Chem. Theor. Comp. 5 (2009) 2108.

[30] A.D. Becke, J. Chem. Phys. 98 (1993) 5648.

[31] C. Lee, W. Yang, R.G. Parr, Phys. Rev. B. 37 (1988) 785.

[32] M.J. Frisch et al, Gaussian 09 Revision A.1, Gaussian Inc. Wallingford CT 2009.

[33] H. B. Schlegel, J. Comput. Chem., 3 (1982) 214-18.

[34] http://www.chem.duke.edu/ yang/Software/softwareNCI.html

[35] A.D. Becke, Phys. Rev. A 38 (1988) 3098.

[36] J.P. Perdew, Unified Theory of Exchange and Correlation Beyond the Local Density Approximation, Akademishe Verlag, Berlin, 1991. 
[37] V.R. Saunders, R. Dovesi, C. Roetti, M. Causá, N.M. Harrison, R. Orlando, C. Zicovich-Wilson, CRYSTAL98 User's Manual, University of Torino, Torino, 1998.

[38] http://www.tcm.phy.cam.ac.uk/ mdt26/crystal.html

[39] M. Prencipe, A. Zupan, R. Dovesi, E. Aprá, V.R. Saunders, Phys. Rev. B 51 (1995) 3391.

[40] A. Otero-de-la-Roza, J. Contreras-Garca, E. R. Johnson (sent)

[41] P. Giannozzi, S. Baroni, N. Bonini, M. Calandra, R. Car, C. Cavazzoni, D. Ceresoli, G. Chiarotti, M. Cococcioni, I. Dabo, et al., J. Phys.: Condens. Matter 21 (2009) 395502.

[42] X. Gonze, J. Beuken, R. Caracas, F. Detraux, M. Fuchs,G. Rignanese, L. Sindic, M. Verstraete, G. Zerah, F. Jollet, et al., Comput. Mater. Sci. 25 (2002) 478.

[43] X. Gonze, Z. Kristallogr. 220 (2005) 558.

[44] G. Kresse, J. Furthuller, Comput. Mat. Sci. 6 (1996) 15.

[45] G. Kresse, J. Furthuller, Phys. Rev. B 54 (1996) 11169.

[46] K. Schwarz, P. Blaha, G. K. H. Madsen, Comput. Phys. Commun. 147 (2002) 71.

[47] K. Schwarz, P. Blaha, Comput. Mater. Sci. 28 (2003) 259.

[48] K. Schwarz, P. Blaha, G.K.H. Madsen, Comput. Phys. Commun. 147 (2002) 71.

[49] K. Dewhurst, S. Sharma, et al., The elk fp-lapw code, http://elk.sourceforge.net/.

[50] V. Luaña, A. Martín Pendás, J.M. Recio, E. Francisco, and M. Bermejo, Comp. Phys. Commun. 77 (1993) 107.

[51] A. Kokalj, J. Molec. Graph. Mod. 17 (1999) 176.

[52] M.A. Spackman, E.N. Maslen, J. Phys. Chem. 90 (1986) 2020.

[53] A. Martín Pendás, V. Luaña, L. Pueyo, E. Francisco, P. Mori-Sánchez, J. Chem. Phys. 117 (2002) 1017

[54] J. Contreras-García, E.R. Johnson, W. Yang, J. Phys. Chem. A 115 (2011) 12983

[55] Y.V. Nelyubina, M.Y. Antipin, K.A. Lyssenko Russ. Chem. Rev. 79 (2010) 167. 
[56] L. Glasser, H.D.B. Jenkins, Chem. Soc. Rev. 34 (2005) 866.

[57] P. Coppens, Annu. Rev. Phys. Chem. 43 (1992) 663.

[58] X. Krokidis, S. Noury, B. Silvi, J. Phys. Chem. A 101 (1997) 7277.

[59] J.A. Wasastjerna, Soc. Sci. Fenn., Commentat. Phys.-Math. 38 (1923) 1.

[60] L. Pauling, J. Am. Chem. Soc. 49 (1927) 765. 